Paul Preisser

Abteilung für Handchirurgie, Plastische und Mikrochirurgie, Zentrum für Schwerbrandverletzte, BG-Unfallkrankenhaus Hamburg

\title{
Operative Behandlung von Pseudarthrosen an Mittelhand und Phalangen
}

rakturen an Mittelhand und Phalan-

\section{Zusammenfassung}

Pseudarthrosen an Mittelhand und Phalangen sind selten. Sie gehen jedoch mit einer erheblich verlängerten Behandlungszeit sowie funktionellen Einbußen einher.In einem 5-Jahres-Zeitraum wurden 47 Pseudarthrosen an Mittelhand und Phalangen operativ versorgt. Es handelte sich in 45 Fällen um Folgezustände nach komplexen Verletzungen mit begleitendem Weichteilschaden, bei 2 Patienten nach primär geschlossenen Frakturen. Nach Ausräumung der Pseudarthrose wurde der resultierende knöcherne Defekt durch autologe Knochentransplantation oder eine Verkürzung überbrückt.Zur Osteosynthese wurden Kirschner-Drähte oder Titanminiimplantate verwendet. Von 45 nachverfolgten Fällen konnten 43 zur knöchernen Abheilung gebracht werden. Bei 11 Patienten waren Nachoperationen erforderlich, davon in 7 Fällen sekundäre Rekonstruktionen zur Funktionsverbesserung. Ein Finger wurde wegen persistierender Pseudarthrose und Funktionslosigkeit amputiert.

\section{Schlüsselwörter}

Pseudarthrosen - Mittelhand · Phalangen . Osteosynthese $\cdot$ Knochentransplantation gen zeigen sowohl bei konservativer als auch bei operativer Frakturbehandlung eine hohe Ausheilungsrate. Die Pseudarthroserate wird in großen Studien mit zwischen 0,5 und mehr als $10 \%$ angegeben, wobei deutlich unterschiedlich zusammengesetzte Patientenkollektive betrachtet wurden. Übereinstimmend wird als wesentlicher Faktor einer fehlenden Knochenbruchheilung eine komplexe Verletzung mit instabiler Fraktur und Weichteilschaden angesehen. Häufig liegt eine unzureichend stabile Osteosynthese vor; zusätzlich können Sehnen, Gefäße oder Nerven mit verletzt sein [2, $3,8,9,10]$. Während einzelne Autoren eine erhöhte Pseudarthroserate nach Osteosynthesen mit Miniplatten und Schrauben beschrieben $[5,11,13]$, gaben andere Autoren eine Verbesserung der Heilungsrate durch weiterentwickelte Miniimplantate an $[1,7]$.

Zur operativen Versorgung der Pseudarthrosen gehören

- eine adäquate Weichteildeckung,

- die vollständige Entfernung des pseudarthrotischen Knochendefekts,

- eine autologe Knochentransplantation sowie

- eine ausreichende knöcherne Stabilität $[6,9]$.

Bei der Wahl des Osteosyntheseverfahrens kommen Platten und Schraubenosteosynthese sowie bei schlechter Weichteildeckung Kirschner-Drähte und intraossäre Drahtnähte in Betracht. Der Fixateur externe hat auch bei der
Versorgung infizierter Pseudarthrosen an der Hand seinen Stellenwert.

\section{Patienten und Methode}

\section{Patienten}

In einem 5-Jahres-Zeitraum zwischen 1995 und 1999 wurden insgesamt $47 \mathrm{Se}$ kundäreingriffe wegen nicht verheilter Frakturen an Mittelhand und Phalangen vorgenommen. Es handelte sich dabei um 45 offene Verletzungen mit Weichteilschaden, davon in 11 Fällen um Folgezustände nach Replantationen und Revaskularisationen. Als Unfallursache standen Säge- und offene Quetschverletzungen im Vordergrund. Wegen unzureichender Weichteildeckung wurden bei 3 Patienten Lappenplastiken im Rahmen vorbereitender Eingriffe vorgenommen. Bestehende tiefe Infektionen bei 5 Patienten erforderten die Einlage von PMMA-Miniketten, bei 3 Patienten zusätzlich eine Sequesterotomie. Immer erfolgte hier eine temporäre Fixierung durch einen Fixateur externe. Nur bei 2 Patienten führte jeweils eine geschlossene Fraktur zur Pseudarthrosenentwicklung, in beiden Fällen nach einer Osteosynthese mit Titanminifragment-

\footnotetext{
Dr. Paul Preisser

Abteilung für Handchirurgie, Plastische und Mikrochirurgie, Zentrum für Schwerbrandverletzte, BG-Unfallkrankenhaus Hamburg, Bergedorfer Straße 10, 21033 Hamburg, E-Mail:P.Preisser@BUK-Hamburg.de, Phone:040-73063946, Fax:040-73062750
} 


\section{P. Preisser}

\section{Operative treatment of nonunions of the metacarpal and phalangeal bones}

\section{Abstract \\ Nonunion is rare in the metacarpals and phalanges. It is followed by functional deficits and a particularly lengthy rehabilita- tion. In a 5-year period 47 cases of such non- union were treated surgically. In 45 cases complex trauma had preceded the non- union, and only 2 patients had had primary closed fractures and titanium miniplate fixa- tion. All patients were treated with internal fixation with K-wires or titanium miniplates plus additional autologous bone transplant or shortening. Among 45 of these patients who were followed up, bony union was archieved in 43; 11 patients had further op- erations, and 7 of these also underwent sec- ondary procedures to improve mobility and function. In 1 patient a finger in which non- union persisted had to be amputated.}

\section{Keywords}

Nonunion - Metacarpal bone · Phalanges Osteosynthesis - Bone graft

\section{Tabelle 1 \\ Unfallursache bei Pseudarthrosen an Mittelhand und Phalangen}

\begin{tabular}{lr} 
Unfallursache & Patientenzahl \\
\hline Kreissäge & 15 \\
Quetschung & 21 \\
Sportverletzung & 2 \\
Verkehrsunfall & 2 \\
Sonstige & 7
\end{tabular}

platte (Tabelle 1). Mittelhand, Grundglied sowie Mittel- und Endglied waren gleichmäßig betroffen (Tabelle 2).

Im Rahmen der Erstversorgung waren in 4 Fällen die Frakturen nach der Wundversorgung primär konservativ behandelt worden. Bei 37 Patienten war eine Osteosynthese oder primäre Arthrodese mit Kirschner-Drähten vorangegangen, in 6 Fällen mit Titanminiimplantaten.

17 Patienten waren im eigenen Haus voroperiert, 30 Patienten wurden nach auswärtiger Erstbehandlung zur Pseudarthrosenversorgung zugewiesen. Der durchschnittliche Abstand zwischen Fraktur und Pseudarthrosenversorgung betrug 241 Tage.

\section{Operationstechnik}

Bei der operativen Versorgung wurde die Pseudarthrose bis in sicher gesund erscheinendes Knochengewebe entfernt. Oft war hierzu die Entnahme eines kompletten Knochensegments notwendig. Sehr gelenknahe oder in die Gelenkfläche hinein reichende Pseudarthrosen wurden durch Resektion der Gelenkflächen zur gleichzeitigen Arthrodesierung behandelt.

Der knöcherne Defekt wurde in 7 Fällen mit einer autologen Spongiosaplastik, in 30 Fällen mit einem korti-

\begin{tabular}{|c|c|}
\hline Lokalisation & Patientenzahl \\
\hline Mittelhand & 10 \\
\hline Grundglied & 15 \\
\hline Mittelglied, Mittelgelenk & 11 \\
\hline Endglied, Endgelenk & 11 \\
\hline
\end{tabular}

kospongiösen Beckenkammspan überbrückt. In 10 Fällen erfolgte die Kontinuitätsherstellung durch eine primäre Verkürzung, sämtlich bei Pseudarthrosen distal des Fingergrundglieds und schlechter Weichteildeckung, in 7 Fällen als Arthrodese des Mittel- oder Endgelenks.

Zur knöchernen Stabilisierung wurde bei allen Patienten, auch bei beruhigter knöcherner Infektion, eine interne Stabilisierung durchgeführt; in 13 Fällen mit einer Titanminifragmentplatte und in 34 Fällen mit Kirschner-Drähten und intraossären Drahtnähten jeweils in Höhe der Osteotomieflächen. Der Kirschner-Draht wurden nach knöcherner Konsolidierung entfernt; intraossäre Drahtnähte und Titanminiimplantate wurden belassen.

Weitere Eingriffe zur Verbesserung der Beweglichkeit wie Tenolysen oder Arthrolysen und Weichteilkorrekturen wurden nach definitiver knöcherner Ausheilung und abgeschlossener Übungsbehandlung durchgeführt.

\section{Ergebnisse}

Bei der Versorgung der 47 posttraumatischen Pseudarthrosen konnte in 43 Fällen eine Konsolidierung ohne weitere Folgeeingriffe erzielt werden; bei $2 \mathrm{~Pa}$ tienten kam es nicht zum knöchernen Durchbau, 2 Patienten konnten nicht nachverfolgt werden.

Neben der Entfernung von Kirschner-Drähten wurden Folgeeingriffe bei 11 Patienten notwendig. Ein Finger wurde wegen fortbestehender Pseudarthrose und Dystrophie sekundär amputiert. Bei 4 Patienten erfolgten Nachoperationen zur Behandlung operativer Frühkomplikationen. Bei 3 Patienten wurden rekonstruktive Maßnahmen am Bewegungsapparat durchgeführt, bei $4 \mathrm{~Pa}$ tienten sekundäre Korrekturen am Weichteilmantel. Bis zum Eintritt der Arbeitsfähigkeit verstrichen von der Fraktur an zwischen 70 und 1266 Tage, im Durchschnitt 333 Tage.

Die funktionellen Ergebnisse variierten in Abhängigkeit von der Ausgangssituation stark. Entscheidend waren neben der Verletzungsschwere die Lokalisation der Pseudarthrose, ferner die Art und der zeitliche Ablauf der operativen Versorgung.

Günstige Ergebnisse hinsichtlich der Beweglichkeit konnten bei der Pseudarthrosenversorgung an der Mittelhand 

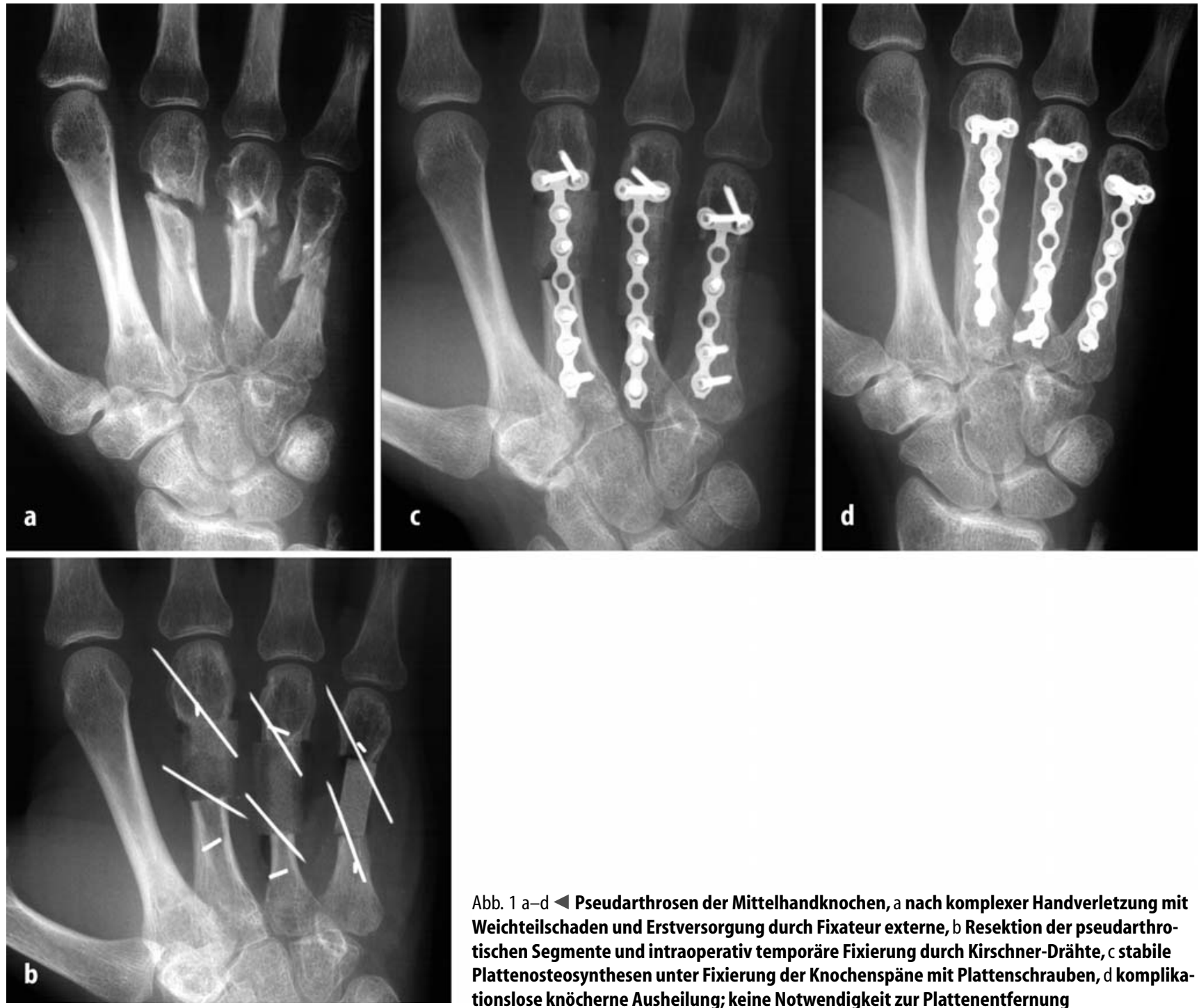

Abb. 1 a-d $<$ Pseudarthrosen der Mittelhandknochen, a nach komplexer Handverletzung mit Weichteilschaden und Erstversorgung durch Fixateur externe, $b$ Resektion der pseudarthrotischen Segmente und intraoperativ temporäre Fixierung durch Kirschner-Drähte, c stabile Plattenosteosynthesen unter Fixierung der Knochenspäne mit Plattenschrauben, $d$ komplikationslose knöcherne Ausheilung; keine Notwendigkeit zur Plattenentfernung

erzielt werden. Im Bereich der Grundglieder wurde nach der Implantation kortikospongiöser Späne jeweils eine deutliche Verschlechterung der Beweglichkeit der distal gelegenen Gelenke bis hin zur Einsteifung beobachtet. So wurde bei Spanaufbau und Osteosynthese in Grundgliedhöhe ein durchschnittlicher aktiver Bewegungsumfang des Mittelgelenks von lediglich $15^{\circ}$, des Endgelenks von $12^{\circ}$ festgestellt. Bei distal des Grundglieds gelegenen Pseudarthrosen war die Beweglichkeit des Grundgelenks gut, die des Mittelgelenks abhängig von der Nähe zur Pseudarthrose und der Schwere des begleitenden Sehnen- und Weichteilschadens.

\section{Diskussion}

Im Gegensatz zu zahlreichen Publikationen zu den Resultaten spezieller Osteosyntheseverfahren existieren nur wenige Studien $\mathrm{zu}$ den Komplikationen der Osteosynthese am Handskelett und nur einzelne Mitteilungen zu den Behandlungsergebnissen der gestörten Knochenbruchheilung. Übereinstimmend wurde von den Autoren ein multifaktorielles Geschehen bei komplexer Handoder Fingerverletzung als ursächlich angegeben $[1,9,14]$. Ob die von einigen Autoren beschriebene erhöhte Komplikationsrate nach Miniplattenosteosynthese und der damit verbundenen früheren Mobilisation und Beübung tatsächlich angenommen werden muss, kann aus den vorliegenden Studien noch nicht eindeutig beantwortet werden. Mit verbesserten Implantaten und zunehmender Erfahrung kann hier in Zukunft jedoch mit einer weiteren Verbesserung der Ergebnisse gerechnet werden. Im eigenen Kollektiv spielt die Miniplattenosteosynthese bei der Pseudarthrosenentstehung lediglich eine untergeordnete Rolle.

Mit der Analyse des eigenen Patientenkollektivs konnte übereinstimmend mit den Resultaten von Jupiter et al. [9] gezeigt werden, dass mit dem beschriebenen Behandlungskonzept eine günstige Prognose hinsichtlich der knöchernen Konsolidierung erzielt werden kann.

Als wesentliches operatives Detail sehen wir die Sanierung einer eventuellen Infektion oder eines schweren Weichteilschadens durch einen vorbe- 

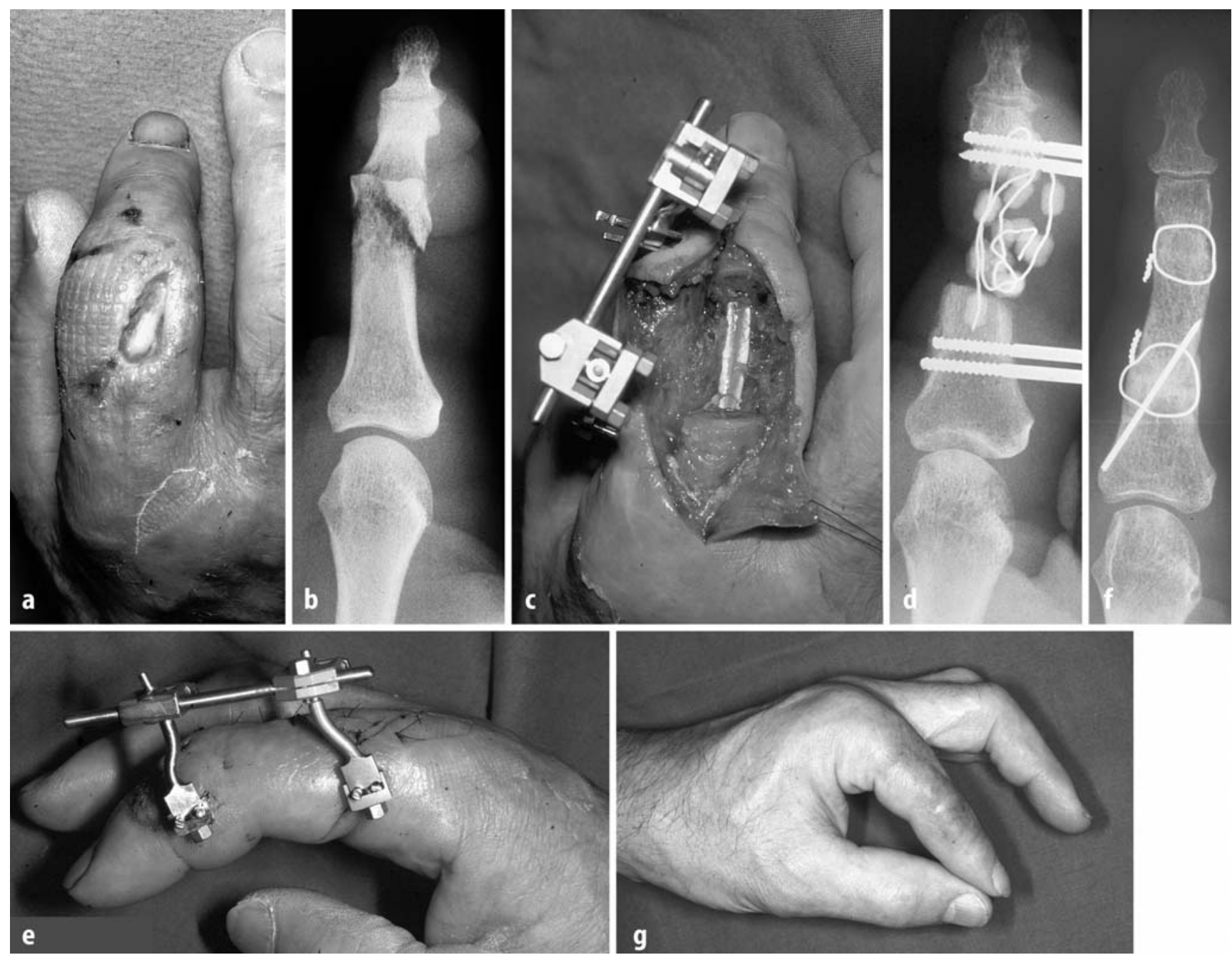

Abb. 2 a-g $\Delta$ Distale Grundgliedfraktur, a, b 0steitis des Zeigefingergrundglieds nach distaler Grundgliedfraktur und Osteosynthese mit Kirschner-Drähten, Weichteildefekt, Nekrose des Grundgliedköpfchens und offenes Mittelgelenk, $c$, d Resektion des distalen Grundglieds und der Mittelgliedbasis mit frei liegenden Beugesehnen am Wundgrund; Einlage einer PMMA-Minikette und Stabilisierung durch Fixateur externe, e Verwendung des Minifixateur externe zur Stabilisierung in funktionsgünstiger Stellung unter Erhalt der ursprünglichen Länge, $\mathrm{f}$ nach Infektberuhigung Mittelgelenkarthrodese unter leichter Verkürzung mit kortikospongiösem Beckenkammspan und Fixierung durch Kirschner-Drähte und intraossäre Drahtnähte, $g$ knöcherne Ausheilung in günstiger Stellung mit stabilen Weichteilverhältnissen

reitenden operativen Eingriff mit radikaler Ausräumung des infizierten Gewebes, einer temporären Stabilisierung und evtl. einer Verbesserung der Weichteilsituation durch eine Hautlappenplastik (Abb. 3). Der Vorteil des zweioder mehrzeitigen Vorgehens wird dabei in der Reduzierung des Heilungsund Infektionsrisikos bei der Spanverpflanzung angesehen.

Als wesentlichen technischen Faktor bei der Pseudarthrosenversorgung sehen wir neben der stabilen Osteosynthese die vollständige Ausräumung der Pseudarthrose und die großzügige Ver- wendung autologer Knochentransplantate. Bei Pseudarthrosen im Bereich von Mittelhand und Fingergrundglied ist die Wiederherstellung der ursprünglichen Länge zum Erhalt der Sehnenspannung notwendig. Der gegenüber der alleinigen Spongiosatransplantation vorrangig verwendete kortikospongiöse Beckenkammspan ermöglicht hier neben der Kontinuitäts- und Längenwiederherstellung eine knöcherne Abstützung und erleichtert damit die stabile Fixierung (Abb. 1).

Bei distal des Grundglieds gelegenen Pseudarthrosen und kurzstreckigem Knochendefekt kann die Spanverpflan- zung mit einer primären Verkürzung vermieden werden. Bei gleichzeitig bestehendem Weichteilschaden wird mit dem Verzicht auf eine vollständige Wiederherstellung der ursprünglichen Länge die Heilung der Weichteile begünstigt.

Die Wahl des Implantats richtet sich nach der Lokalisation der Pseudarthrose und nach der Qualität der Weichteilbedeckung. In funktioneller Sicht bieten stabile Osteosynthesen mit Titanminiimplantaten Vorteile. Mit einer frühfunktionellen Nachbehandlung können ein durch Adhäsionen der Streckaponeurose bedingtes Streckdefizit oder die 


\section{Fehlheilungen}
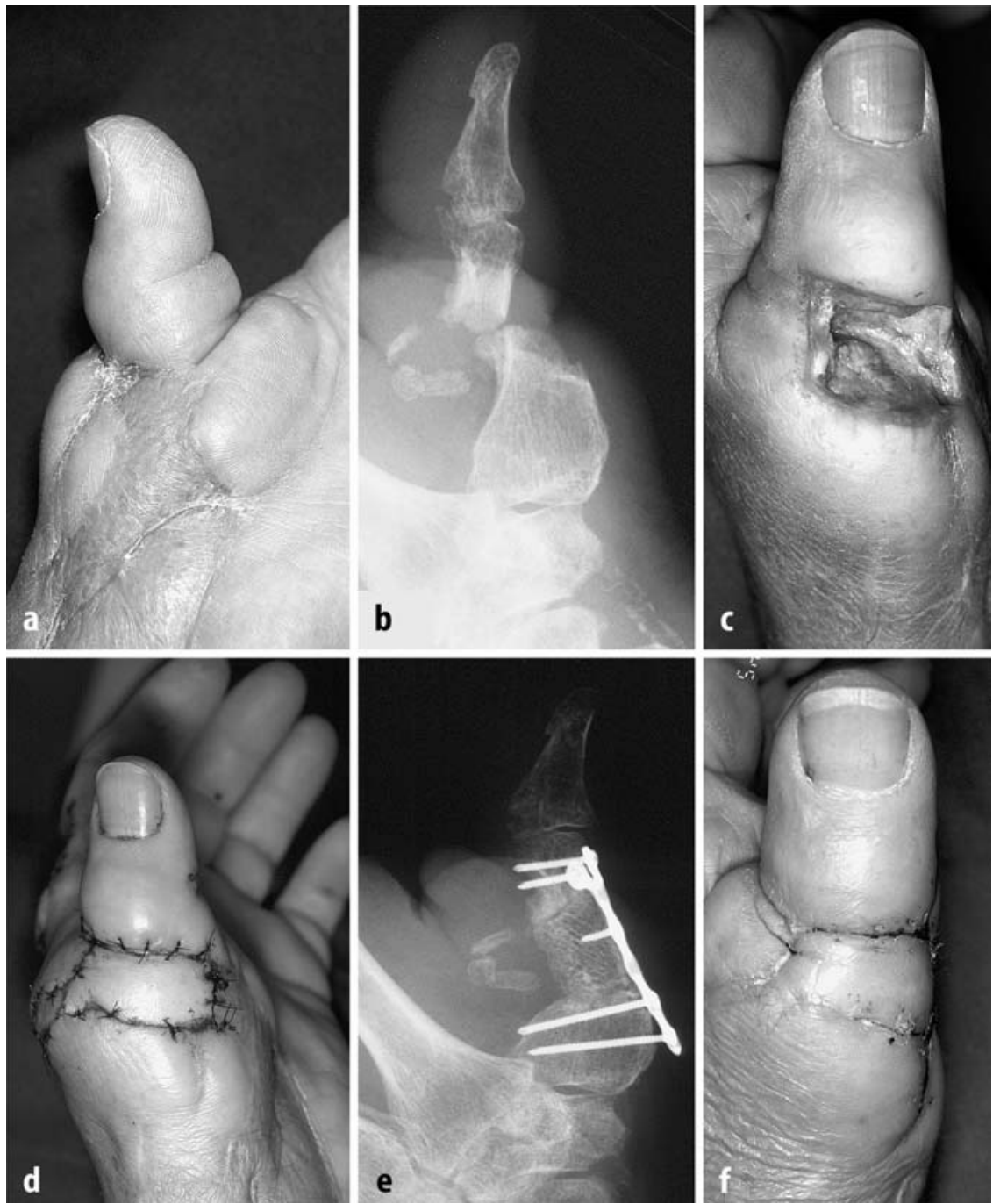

Abb. 3 a-f $\Delta$ Instabile Pseudarthrose nach Daumenquetschung, a, b mit Grundgelenkzerstörung und schwerem Weichteilschaden, intakte Sensibilität beim Fehlen von langer Beugesehne und Streckaponeurose in Endgelenkhöhe, $c, d$ Narbenexzision, Entfernung der Pseudarthrose, Einlage einer PMMA-Minikette und temporäre interne Fixierung durch gekreuzte Kirschner-Drähte, Weichteilsanierung durch Insellappen von der Streckseite des Zeigefingers, $\mathrm{e}, \mathrm{f}$ Pseudarthrosensanierung mit kortikospongiösem Beckenkammspan und Miniplattenosteosynthese bei stabilen Weichteilverhältnissen

Einsteifung der distal der Pseudarthrose gelegenen Gelenke vermieden werden. Wichtigste Voraussetzungen für die Verwendung von Miniplatten sind eine suffiziente Weichteildeckung und ausreichend große knöcherne Fragmente.

Bei distal des Fingergrundglieds gelegenen Pseudarthrosen ist aufgrund der Weichteilbedeckung eine Fixierung mit Schrauben und Platten oft problematisch. Mit der Verwendung von Kirschner-Drähten und intraossären Drahtnähten können hier der notwendige operative Zugang begrenzt und eine zusätzliche Weichteiltraumatisierung vermieden werden (Abb. 2).
Der Fixateur externe wurde im eigenen Kollektiv im Rahmen vorbereitender Eingriffe zur Sanierung einer posttraumatischen Infektion sowie bei ausgedehnten knöchernen Defekten nach posttraumatischer Infektion zur vorübergehenden Stabilisierung eingesetzt und ist bei dieser Indikation unverzichtbar (Abb. 2). Bei der nachfolgenden definitiven Pseudarthrosenversorgung wurde eine interne Stabilisierung vorgenommen. Gegen die Verwendung des Fixateur externe zur definitiven Pseudarthrosenbehandlung sprechen die notwendige lange Tragedauer sowie die bestehende Infektionsgefahr bei der unvermeidlichen Platzierung der Pins in Gelenknähe sowie in Nachbarschaft der Transplantate.

Während die Prognose hinsichtlich der knöchernen Heilung gut ist, ist das funktionelle Ergebnis nach erfolgreicher knöcherner Heilung von zahlreichen Faktoren abhängig. Wesentlich sind die Lokalisation und der Umfang des knöchernen Defekts, die Art des Weichteilschadens und der Schädigung von Sehnen, Nerven und Gefäßen [4, 9, 14]. Bei Pseudarthrosen im Phalangenbereich muss bei einem großen Anteil der $\mathrm{Pa}$ tienten mit erheblichen Bewegungseinschränkungen der Fingergelenke gerechnet werden. In besonderem Maß betroffen sind dabei die Pseudarthrosen des Fingergrundglieds, bei denen durch Adhäsionen der Streckaponeurose eine deutliche Einschränkung der Beweglichkeit von Mittel- und Endgelenk erwartet werden muss. Ob mit der Einführung von Titanminiimplantaten und einer stabilen Osteosynthese eine Besserung der Funktion erzielt werden kann, kann aus den bisherigen Ergebnissen nicht eindeutig beantwortet werden und bleibt weiter abzuwarten.

Sekundäre Rekonstruktionen an Sehnen und Gelenken erfordern eine frühe postoperative Mobilisation zum Erhalt der Beweglichkeit. Diese können mit Aussicht auf Erfolg erst nach vollständiger Konsolidierung und Narbenlockerung durchgeführt werden. Neben der weiteren Verlängerung der Rehabilitationszeit muss häufig mit funktionellen Einbußen gerechnet werden.

\section{Hinweise für die Praxis}

Folgendes ist bei der operativen Behandlung von Pseudarthrosen an Mittelhand und Phalangen zu beachten:

- Die Sanierung einer Pseudarthrose an Mittelhand oder Phalangen erfordert die vollständige Entfernung des pseudarthrotischen Gewebes und eine Überbrückung des resultierenden Knochendefekts durch autologe Knochen(span)plastik oder Verkürzung.

- $\quad$ Eine vorbestehende Infektion oder Weichteilschaden sollten im Rahmen eines mehrzeitigen Vorgehens durch einen vorbereitenden Eingriff saniert werden. 
- Lokalisation der Pseudarthrose, Knochensubstanz und Weichteilbedeckung bestimmen die Wahl des 0 steosyntheseverfahrens. Eine stabile Osteosynthese mit Miniplatten ermöglicht eine frühe funktionelle Nachbehandlung. Kirschner-Drähte haben weiterhin ihre Indikation bei kleinen gelenknahen Fragmenten und bei distal gelegenen Pseudarthrosen mit knapper Weichteilbedeckung.

- Sekundäre Rekonstruktionen an Sehnen und Gelenken sind erst nach vollständiger knöcherner Konsolidierung Erfolg versprechend.

- Angesichts der vielfältigen, zur Pseudarthrose führenden Verletzungsformen ist ein individuelles Vorgehen notwendig. Dies betrifft die Art und die zeitliche Sequenz der notwendigen operativen Maßnahmen, die Wahl des entsprechenden Osteosyntheseverfahrens sowie die postoperative Nachbehandlung und Rehabilitation.

\section{Literatur}

1. Bartelmann U, Kotas J, Landsleitner B (1997) Ursachen von Nachoperationen nach Osteosynthesen von Finger- und Mittelhandfrakturen. Handchir Plast Chir Mikrochir 29:204208

2. Barton NJ (1979) Fractures of the shafts of the phalanges of the hand. Hand 11:119-133

3. Borgeskov S (1967) Conservative therapy for fractures of the phalanges and metacarpals. Acta Chir Scand 133:123-130

4. Duncan RW, Freeland AE, Jabaley ME, Meydrech EF (1993) Open hand fractures: an analysis of the recovery of active motion and of complications.J Hand Surg [Am] 18A:387-394

5. Fusetti C, Meyer H, Borisch N, Stern R, Santa DD, Papaloizos M (2002) Complications of plate fixation in metacarpal fractures.J Trauma 52: 535-539

6. Green DP (1986) Complications of phalangeal and metacarpal fractures. Hand Clin 2:307328

7. Helaly $\mathrm{P}(1991)$ Vereinfachung des Osteosyntheseverfahrens am Handknochen durch neuentwickelte Miniosteosynthesen.Z Unfallheilkd Versicherungsmed 84: 140-147

8. Ip WY, Ng KH, Chow SP (1996) A prospective study of 924 digital fractures of the hand. Injury 27:279-285
9. Jupiter JB, Koniuch MP, Smith RJ(1985) The management of delayed union and nonunion of the metacarpals and phalanges.J Hand Surg Am 10A:457-466

10. Oostterom FJT van, Brete GJV, Ozdemir C, Hovius SER (2001) Treatment of phalangeal fractures in severely injured hands.J Hand Surg [Br] 26B: 108-111

11. Page SM, Stern PJ (1998) Complications and range of motion following plate fixation of metacarpal and phalangeal fractures.J Hand Surg [Am] 23A:827-832

12. Schwarz N, Eben K (1980) Pseudarthrosen an Finger und Mittelhandknochen. Hefte Unfallheilkd 141:180-188

13. Stern PJ, Wiesner MJ, Reilly DG (1987) Complications of plate fixation in the hand skeleton. Clin Orthop 214:59-65

14. Wray RC Jr, Glunk R (1989) Treatment of delayed union, nonunion, and malunion of the phalanges of the hand. Ann Plast Surg 22: 14-18 